\title{
PEGylated ofloxacin nanoparticles render strong antibacterial activity against many clinically important human pathogens
}

\author{
Gregory Marslin a,1, Ann Mary Revina ${ }^{\mathrm{b}, \mathrm{c}, 1}$, Vinoth Kumar Megraj Khandelwal ${ }^{\mathrm{d}}$, \\ Krishnamoorthy Balakumar ${ }^{\mathrm{e}}$, Caroline J. Sheeba ${ }^{\mathrm{b}, \mathrm{c}, \mathrm{f}, \mathrm{g}, 2}$, Gregory Franklin ${ }^{\mathrm{a}, *, 2}$ \\ a Centre for the Research and Technology of Agro-Environment and Biological Sciences (CITAB), University of Minho, Portugal \\ ${ }^{\mathrm{b}}$ Life and Health Sciences Research Institute (ICVS), School of Health Sciences, University of Minho, Portugal \\ ' ICVS/3B's - PT Government Associate Laboratory, Braga/Guimarães, Portugal \\ d Consorzio Mario Negri Sud, Department of Translational Pharmacology, Santa Maria Imbaro, Italy \\ e Department of Pharmaceutics, PSG College of Pharmacy, Coimbatore, Tamilnadu, India \\ ${ }^{\mathrm{f}}$ Regenerative Medicine Program, Departamento de Ciências Biomédicas e Medicina, Universidade do Algarve, Faro, Portugal \\ g IBB-Institute for Biotechnology and Bioengineering, Centro de Biomedicina Molecular e Estrutural, Universidade do Algarve, Faro, Portugal
}

\section{A R T I C L E I N F O}

\section{Article history:}

Received 9 February 2015

Received in revised form 22 April 2015

Accepted 23 April 2015

Available online 4 May 2015

\section{Keywords:}

Antibiotic resistance

Ofloxacin

mPEG-PLGA diblock Co-polymer

Nanoparticles

Antibacterial

Bacterial uptake

\begin{abstract}
A B S T R A C T
The rise of bacterial resistance against important drugs threatens their clinical utility. Fluoroquinones, one of the most important classes of contemporary antibiotics has also reported to suffer bacterial resistance. Since the general mechanism of bacterial resistance against fluoroquinone antibiotics (e.g. ofloxacin) consists of target mutations resulting in reduced membrane permeability and increased efflux by the bacteria, strategies that could increase bacterial uptake and reduce efflux of the drug would provide effective treatment. In the present study, we have compared the efficiencies of ofloxacin delivered in the form of free drug (OFX) and as nanoparticles on bacterial uptake and antibacterial activity. Although both poly(lactic-co-glycolic acid) (OFX-PLGA) and methoxy poly(ethylene glycol)-b-poly(lactic-co-glycolic acid) (OFX-mPEG-PLGA) nanoformulations presented improved bacterial uptake and antibacterial activity against all the tested human bacterial pathogens, namely, Escherichia coli, Proteus vulgaris, Salmonella typhimurium, Pseudomonas aeruginosa, Klebsiella pneumoniae and Staphylococcus aureus, OFX-mPEG-PLGA showed significantly higher bacterial uptake and antibacterial activity compared to OFX-PLGA. We have also found that mPEG-PLGA nanoencapsulation could significantly inhibit Bacillus subtilis resistance development against OFX.
\end{abstract}

(C) 2015 Elsevier B.V. All rights reserved.

\section{Introduction}

The fluoroquinolone derivative ofloxacin(OFX) is a racemic mixture, which consists of $50 \%$ levofloxacin (the biologically active component) and $50 \%$ of its enantiomer dextrofloxacin. It inhibits bacterial multiplication by interacting with type II topoisomerases (i.e. gyrase and topo IV) that are involved in the replication and repair of bacterial DNA [1]. Since type II topoisomerases are found in most of the bacteria, ofloxacin serves as a broad-spectrum antibiotic [2-4]. Ofloxacin is currently used in the treatment of pneumonia, chronic bronchitis, urinary tract infection and certain sexually

\footnotetext{
* Corresponding author. Tel.: +351 253604865; fax: +351 253604809

E-mail address: franklin@bio.uminho.pt (G. Franklin).

1 Co-first authors.

2 These authors contributed equally.
}

transmitted diseases [5-7]. However, several disease-causing bacteria have already gained resistance against this class of antibiotics, invalidating its clinical use [8,9]. Hence, delivery strategies of antibiotics with improved efficacy and avoidance of resistance are highly desirable.

In order to develop fluoroquinolone resistance, Gram-negative bacteria adopts mechanisms such as, decreased outer membrane permeability to limit the cellular entry and increased efflux pumps activity to extrude quinolones that gained access to the cytoplasmic membrane [10]. In this context, altering the level of porin1a $(\mathrm{OmpF})$ in the outer membrane, a protein that is known to regulate membrane permeability and passive diffusion, has been shown to confer antimicrobial resistance in many Gram-negative bacteria [11]. Whereas, Gram-positive bacteria (example: Bacillus subtilis) develop drug resistance by expressing drug transporters (bmr) and by targeted mutation mechanisms (gyrA and parC) in response to antibiotic pressure [12]. Hence, exploring the possibility of 
improving the bacterial uptake of fluoroquinolone and inhibiting its efflux system is a rational strategy to challenge bacterial resistance.

Utilization of antibacterial nanoparticles is an appropriate and attractive way to fight bacterial resistance. Nanoparticles are submicron-size carrier systems composed of natural or synthetic polymers with the size range of $10-1000 \mathrm{~nm}$ in which the drug may be dissolved, entrapped, encapsulated, or attached [13]. Poly(D,L-lactic-co-glycolic acid) (PLGA) is a widely used polymer for nanoencapsulation and is considered highly safe for systemic administration because it is completely metabolized by the body and approved by the FDA [14]. The final degradation products such as lactic and glycolic acids of this polymer are also totally safe, as they are either excreted by the kidney or enter the Krebs' cycle and eventually eliminated as carbon dioxide and water [15]. The other polymer used in this study is methoxy poly(ethylene glycol)b-poly(lactic-co-glycolic acid) (mPEG-PLGA), which is a diblock copolymer, containing a methoxy end-capped hydrophilic PEG block and a hydrophobic block composed of PLGA. Since PEG has the ability to bind DNA [16,17] and block drug efflux pumps [18,19], we hypothesize that by utilizing mPEG-PLGA nanoparticles, it is possible to improve the efficiency of OFX by delivering it at the site of action while inhibiting its extrusion.

To test this hypothesis, we compared the efficacy of PLGA and mPEG-PLGA nanoparticles loaded with OFX on in vitro bacterial uptake and antibacterial activity. Based on the results obtained in the present study, we conclude that MPEG-PLGA encapsulated OFX nanoparticles significantly improves bacterial uptake and bactericidal activity. Notably, this nanoformulation also delayed the development of bacterial resistance, compared to the free drug and PLGA nanoformulation. The possible role of PEG in bacterial uptake and inhibition of bacterial resistance is discussed.

\section{Materials and methods}

\subsection{Chemicals and reagents}

Ofloxacin (OFX) was obtained from Orchid Chemicals and Pharmaceuticals (Chennai, India). Poly(D,L-lactic-co-glycolide) (PLGA; molar ratio of 50:50, MW - 50,000), monomethoxy poly(ethylene glycol) (mPEG, MW - 2000), Poly(vinyl alcohol) (PVA) and acetic acid were purchased from Sigma-Aldrich (USA). Sodium hydroxide, phosphate buffer, potassium dihydrogen phosphate, methanol and acetone were acquired from Merck Laboratory Reagents (Germany). All reagents used in this study were analytical grade.

\subsection{Preparation of ofloxacin nanoparticles}

Initially, mPEG-PLGA copolymer was prepared by ring-opening polymerization of PLGA and MPEG in the presence of stannous octanoate as a catalyst. Briefly, $5 \mathrm{~g}$ of PLGA, $5 \mathrm{~g}$ of $\mathrm{mPEG}$, and catalyst $(0.1 \%)$ were added into a $50 \mathrm{ml}$ dried glass reactor. The mixture was heated to reflux with magnetic stirring under a flow of nitrogen for $18 \mathrm{~h}$ and then cooled to room temperature. The reaction product was dissolved in chloroform, and precipitated with an excess of methanol/diethyl ether to obtain a white emulsion. After the evaporation of solvent from emulsion, the resulting aqueous solution was lyophilized to yield the product as a white powder [20].

OFX encapsulated mPEG-PLGA (OFX-mPEG-PLGA) and PLGA (OFX-PLGA) nanoparticles were prepared by emulsion solvent evaporation method [21]. Briefly, $100 \mathrm{mg}$ of mPEG-PLGA or PLGA and $10 \mathrm{mg}$ of OFX were dissolved in $5 \mathrm{ml}$ of chloroform (organic phase). This solution was maintained covered on an ice bath to avoid evaporation and added drop by drop to $50 \mathrm{ml}$ aqueous phase containing 2\% PVA while homogenizing at 18,000 rpm (IKAT 25
Ultra Turrax $^{\circledR}$ homogenizer). After removing chloroform from the mixture by magnetic stirring at room temperature, the suspension was centrifuged at $20,000 \mathrm{rpm}$ for $15 \mathrm{~min}$ to pellet down the nanoparticles. Nanoparticle pellet was washed three times with ultra-pure water to remove any free drug. After confirming that the wash solution is free of drug, nanoparticles were freeze-dried and stored at $4{ }^{\circ} \mathrm{C}$. Coumarin 6-loaded PLGA and mPEG-PLGA nanoparticles (COU-PLGA and COU-mPEG-PLGA) were prepared following the same procedure to investigate bacterial uptake of nanoparticles by flow cytometry and fluorescence microscopy.

\subsection{Physicochemical characterization}

Particle size, polydispersive index and zeta potential of nanoparticles were determined using a Zetasizer (Zetasizer nano ZS, Malvern Instruments, UK). Morphological characteristics of the nanoparticles were confirmed by transmission electron microscopy (TEM, JEOL-1400, 902A Jeol Ltd.) after negative staining with sodium phosphotungstate solution $(2 \%, \mathrm{w} / \mathrm{w})$. The surface morphology of nanoparticle was studied by atomic force microscopy (AFM). AFM images were recorded using tapping mode in air on a Multi-mode Nanoscope Ila instrument equipped with a J scanner (Veeco Instruments, Santa Barbara, USA) and silicon nanoprobes (NCHV, Veeco). NanoScope software version $4.43 \mathrm{r} 8$ was used for image processing.

\subsection{Drug content and encapsulation efficiency}

Briefly, $20 \mathrm{mg}$ of drug loaded nanoparticles was dissolved in $2 \mathrm{ml}$ of dichloromethane. To this, $5 \mathrm{ml}$ of phosphate buffered saline (PBS, pH 7.4) was added, thoroughly mixed and centrifuged at $10,000 \mathrm{rpm}$ to pellet the debris. Absorbance of the aqueous phase was read in a UV-vis spectrophotometer (Shimadzu UV-1700) at $294 \mathrm{~nm}$ using PBS as blank to determine the drug content from a standard graph as described before [22]. The percentage of encapsulation efficiency (EE\%) and drug content (DC\%) of nanoparticles was determined using the following formula:

$\mathrm{EE} \%=\frac{\text { Weight of encapsulated drug }}{\text { Weight of drug used }} \times 100$

DC\% $=\frac{\text { Weight of encapsulated drug }}{\text { Weight of nanoparticles }} \times 100$

\subsection{In vitro drug release}

OFX loaded PLGA and mPEG-PLGA nanoparticles were tested for in vitro drug release. Briefly, $10 \mathrm{mg}$ of each lyophilized samples were suspended in $2 \mathrm{ml}$ of PBS and transferred into a dialysis bag (MWCO 12,000-14,000 Da). The dialysis bag was then placed into a $100 \mathrm{ml}$ bottle containing $50 \mathrm{ml}$ of PBS that was stirred at $100 \mathrm{rpm}$ at $37^{\circ} \mathrm{C}$. From the bottle, $1 \mathrm{ml}$ sample was withdrawn for every $1 \mathrm{~h}$ interval until $36 \mathrm{~h}$. After each withdrawal, $1 \mathrm{ml}$ of PBS was added to compensate the withdrawn sample and to maintain the original volume $[15,23]$. Drug in the collected samples were analyzed for drug content.

\subsection{Bacterial uptake of nanoparticles}

Flow cytometry analysis and confocal microscopy were performed to study the bacterial uptake of MPEG-PLGA and PLGA nanoparticles. Briefly, $0.5 \mu \mathrm{g}$ of each COU-PLGA and COU-mPEGPLGA nanoparticles were added to $1 \mathrm{ml}$ of $E$. coli culture at exponential phase. After $2 \mathrm{~h}$ of co-incubation, bacteria were pelleted down by centrifugation $(8000 \mathrm{rpm})$. To get rid of free 
nanoparticles, the bacterial pellet was washed several times with sterile distilled water. After confirming by fluorescent microscopy that there is no nanoparticle left in the supernatant, bacterial pellet was resuspended in $1 \mathrm{ml}$ of sterile distilled water. The fluorescent intensity of bacterial cells was observed in an Epics ${ }^{\circledR}$ XLTM cytometer (Beckman Coulter) equipped with an argon-ion laser emitting a $488 \mathrm{~nm}$ beam at $15 \mathrm{~mW}$. Green fluorescence was collected through a $488 \mathrm{~nm}$ blocking filter, a $550 \mathrm{~nm}$ long-pass dichroic and a $225 \mathrm{~nm}$ band-pass filter. Approximately 20,000 cells of each sample were analyzed. Data were analyzed and histograms were made using Flowing Software (version 2.5). In addition, to understand the localization of nanoparticles inside the bacteria, bacterial cells were observed under confocal microscope equipped for photography and images were taken.

\subsection{Quantification of OFX accumulation in E. coli}

Drug accumulation in bacteria was quantified by the method described by Furneri et al. [5] with modification. Briefly, $200 \mathrm{ml}$ of $E$. coli culture at stationary phase $(1.3 \mathrm{OD}$ at $600 \mathrm{~nm}$ ) was pelleted down and gently re-suspended in $20 \mathrm{ml}$ PBS. The bacterial suspension was equilibrated at $37^{\circ} \mathrm{C}$ with agitation and $20 \mu \mathrm{g} / \mathrm{ml}$ OFX or equivalent nanoformulations was added. At pre-determined time intervals, $0.5 \mathrm{ml}$ bacterial suspension was withdrawn and transferred to Eppendorf tubes containing $1 \mathrm{ml}$ of chilled PBS, pelleted by centrifugation at $8000 \mathrm{rpm}$ at $4{ }^{\circ} \mathrm{C}$. After washing with PBS for 3 times, the pellet was resuspended in $1 \mathrm{ml}$ of $0.1 \mathrm{M}$ glycine hydrochloride ( $\mathrm{pH} 3$ ). After overnight digestion, lysate was centrifuged to pellet the debris and the clear supernatant was analyzed for OFX content as mentioned in Section 2.4.

\subsection{Antibacterial activity of OFX loaded PLGA and mPEG-PLGA nanoparticles}

\subsubsection{E. coli growth kinetics}

Initially, E. coli growth kinetics was measured in the presence of OFX in the form of free drug and nanoparticles to understand their effect on bacterial multiplication. For this, equivalent concentration of OFX in the free drug form and nanoparticles (OFX-PLGA and OFX-mPEG-PLGA) were added to $50 \mathrm{ml}$ of Luria-Bertani (LB) medium. By adding $100 \mu \mathrm{l}$ inoculum, cultures were initiated and maintained at $37^{\circ} \mathrm{C}$ with continuous agitation at $200 \mathrm{rpm}$. Controls such as water (equal to the volume of OFX), empty mPEG-PLGA and empty PLGA respective to the concentration of drug-loaded nanoparticles were maintained. Samples $(1 \mathrm{ml})$ were taken after different time intervals and the OD was read at $600 \mathrm{~nm}$ using LB medium as blank.

\subsubsection{Antimicrobial activity against human pathogens}

Antimicrobial activity of nanoformulations was tested against many important human pathogenic bacteria (E. coli, Pseudomonas aeruginosa, Proteus vulgaris, Salmonella typhimurium, Klebsiella pneumoniae and Staphylococcus aureus). Briefly, $25 \mu \mathrm{g} / \mathrm{ml}$ equivalent OFX in the form of nanoparticles or free drug were added to overnight bacterial broth. Corresponding water control and empty nanoparticles (PLGA and mPEG-PLGA) control were also maintained. After $24 \mathrm{~h}$, cultures were serially diluted and spread on LB plates. After 2 days of incubation at $37^{\circ} \mathrm{C}$, the number of colony forming units (CFU) were counted and antibacterial activity was calculated as $\Delta \log _{10} \mathrm{CFU} / \mathrm{ml}$ using the formula: $\Delta\left(\log _{10} \mathrm{CFU} / \mathrm{ml}\right)=\log _{10} \mathrm{CFU} / \mathrm{ml}$ (control) $-\log _{10} \mathrm{CFU} / \mathrm{ml}$ (treatment).

\subsubsection{Bacterial resistance development against OFX}

In order to clearly differentiate the effect of nanoformulations from the free drug, we used $B$. subtilis to test the development of resistance against OFX because this bacterium suffered bacteriostatic and bactericidal effect when treated with OFX-PLGA and OFX-mPEG-PLGA, respectively, but did not display any effect against free OFX (Supplementary data S1). Moreover, B. subtilis has been robustly characterized for the presence of potential drug efflux transporters and MDR-like transporters [12,24], making it a valuable tool to study the development of drug resistance.

Agar well diffusion method was used. Briefly, $100 \mu$ l of $B$. subtilis culture was spread on LB plates. Three wells were made in each plate with the help of sterile cut pipet tips. To each well, $25 \mu \mathrm{g}$ drug equivalent nanoparticles or free drug was introduced. Culture plates were incubated at $37^{\circ} \mathrm{C}$ and the inhibition zones were observed for the development of colonies after 12, 24, 36 and $60 \mathrm{~h}$. To check whether nanoparticles could diffuse in the semisolid medium, COU-PLGA and COU-mPEG-PLGA loaded wells were tracked by fluorescent microscopy.

\subsection{Statistical analysis}

Data were analyzed by Graph Pad Prism6 software (GraphPad, USA). Results are expressed as the means of three replications \pm standard deviation (SD). Statistical significance between treatments was analyzed by one-way ANOVA followed by Bonferroni's Multiple Comparison Test.

\section{Results}

\subsection{Characterization of mPEG-PLGA copolymer}

mPEG-PLGA copolymer was synthesized from lactide and glycolide in the presence of mPEG2000; whose hydroxyl group could initiate the ring opening polymerization of lactide and glycolide. Synthesized copolymer mPEG-PLGA was characterized by 1 HNMR spectra (Supplementary data S3). The large peak at $\delta 3.61 \mathrm{ppm}$ in 1HMNR of mPEG-PLGA (S3.1A) corresponds to the methylene protons of mPEG (S3. 1C), compared to PLGA (S3. 1B), while the integrated signals around $\delta 1.56 \mathrm{ppm}(\mathrm{S} 3.1 \mathrm{~A})$ are due to the methyl protons of the $\mathrm{D}$ - and L-lactic acid repeat units. The characteristic peaks of lactic acid $\mathrm{CH}$ protons at $\delta 5.2 \mathrm{ppm}$ (S3.1A) and glycolic acid $\mathrm{CH} 2$ protons at $\delta 4.8 \mathrm{ppm}(\mathrm{S} 3.1 \mathrm{~A})$ confirm the successful synthesis of mPEG-PLGA copolymer.

\subsection{Characteristics of nanoparticles}

As seen in the TEM image, both OFX-PLGA and OFX-mPEGPLGA nanoparticles exhibited a smooth spherical shape (Fig. 1A and B). AFM analyses revealed that although OFX-PLGA nanoparticles were heterogeneous in their size (Fig. 1C), OFX-mPEG-PLGA nanoparticles are uniformly sized (Fig. 1D), Comparatively, PLGA based nanoparticles were larger and had a higher zeta potential than mPEG-PLGA based nanoparticles (Supplementary data S2). No aggregation or adhesion was observed in both of these nanoparticles.

\subsection{Bacterial uptake of nanoparticles}

No fluorescence was detected in the histogram of control E. coli cells (Fig. 2A). On the other hand, E. coli cells treated with COU-PLGA and COU-mPEG-PLGA nanoparticles showed significant fluorescence in the flow cytometry histogram (Fig. 4B and C). Histograms further revealed that COU-mPEG-PLGA nanoparticles were more efficiently up taken (94\%, Fig. 2C) than COU-PLGA nanoparticles (71\%, Fig. 2B).

To further characterize the internalization of nanoparticles by E. coli, COU-mPEG-PLGA and COU-PLGA treated bacterial samples were analyzed by confocal microscopy. A bright field image of $E$. 


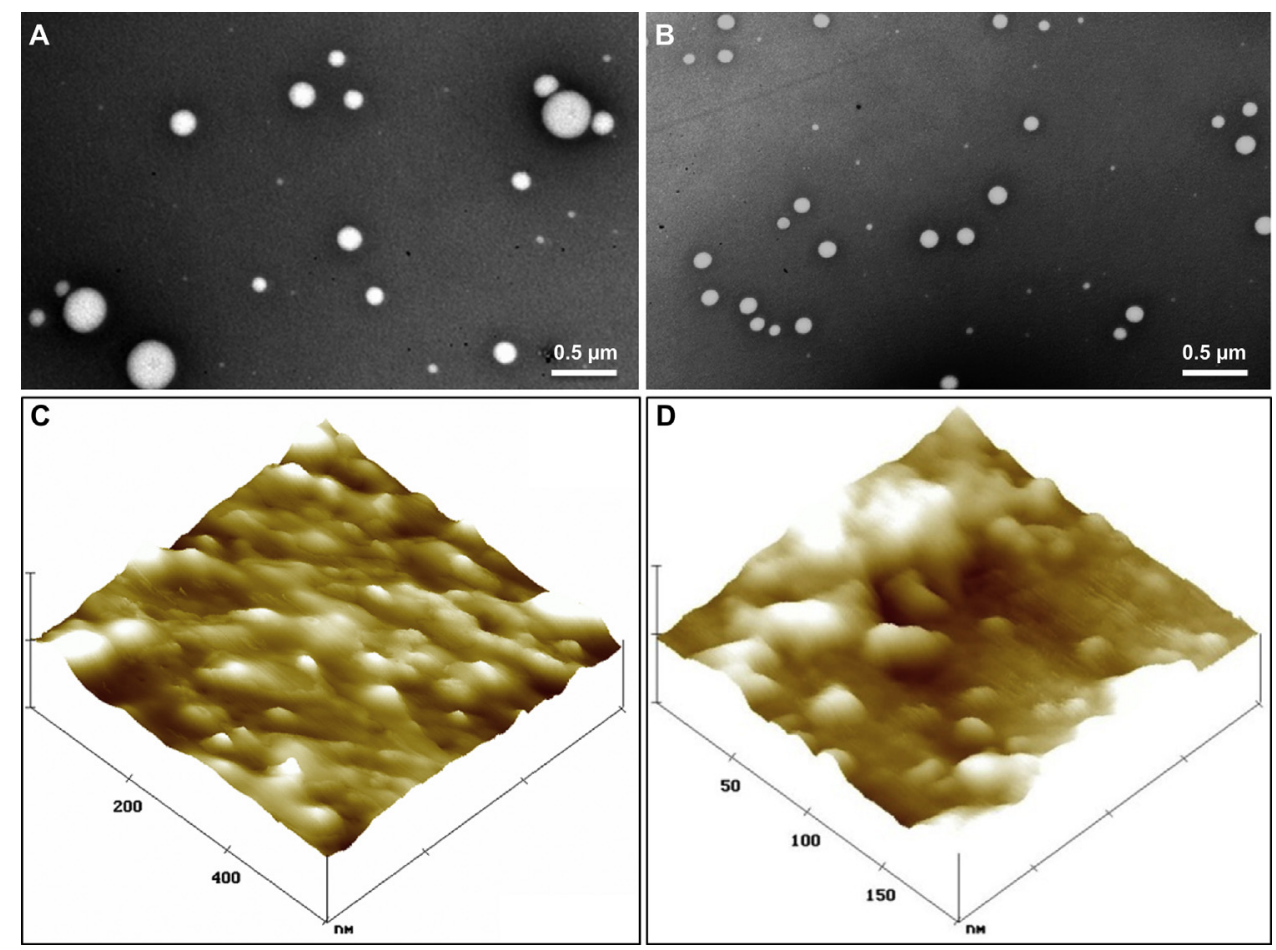

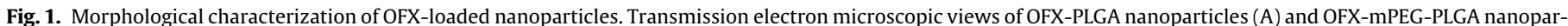
ticles (B) at 30,000 × magnification, Atomic force microscopic 3D images of OFX-PLGA nanoparticles (C) and OFX-mPEG-PLGA nanoparticles (D).

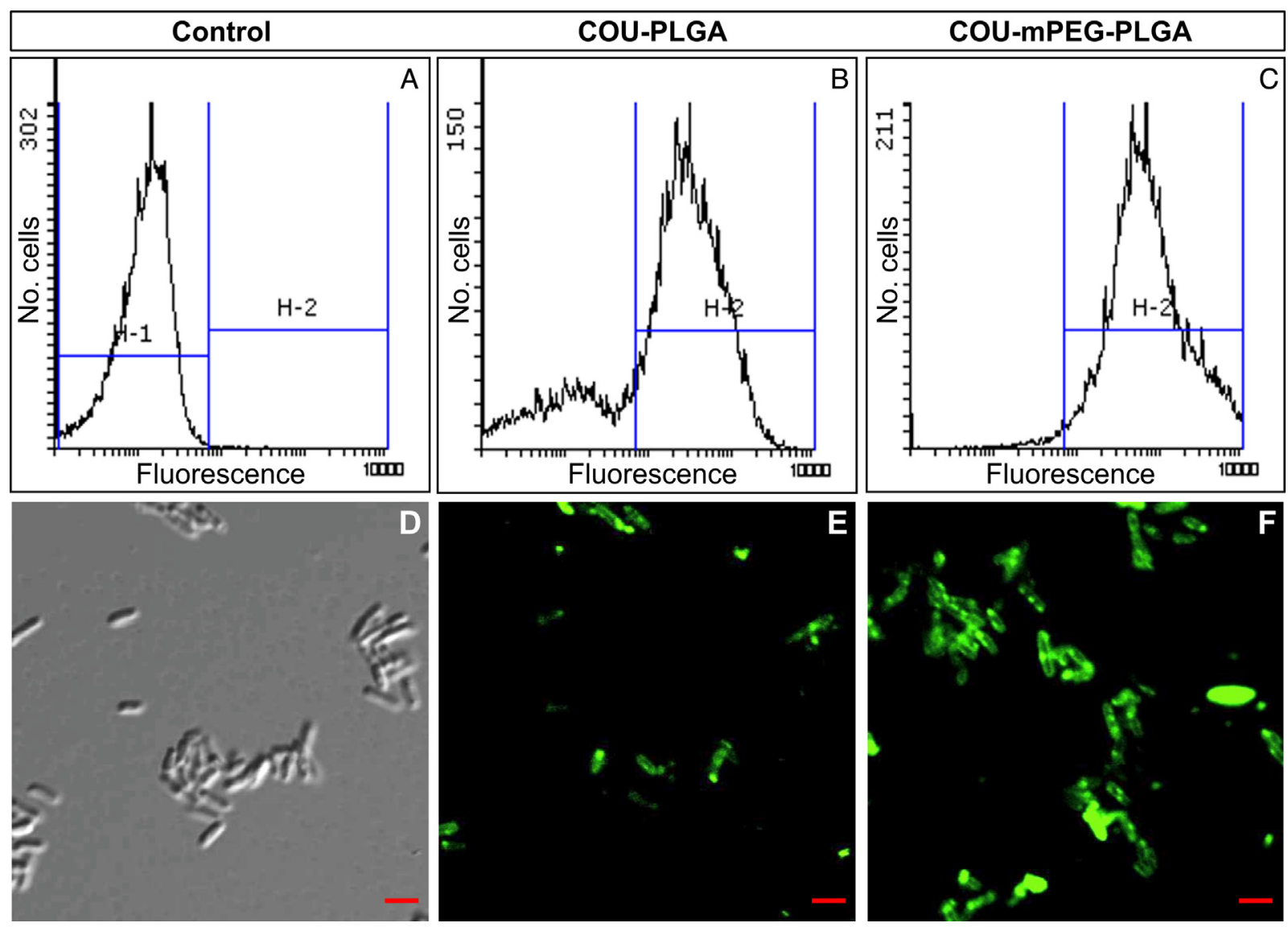

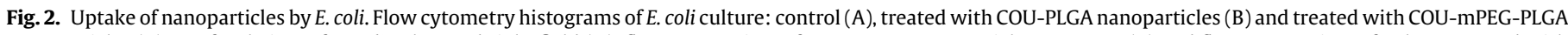

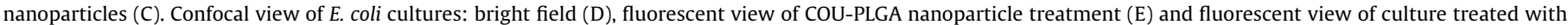
COU-mPEG-PLGA nanoparticles (F). Scale bar $=2 \mu \mathrm{m}$. 

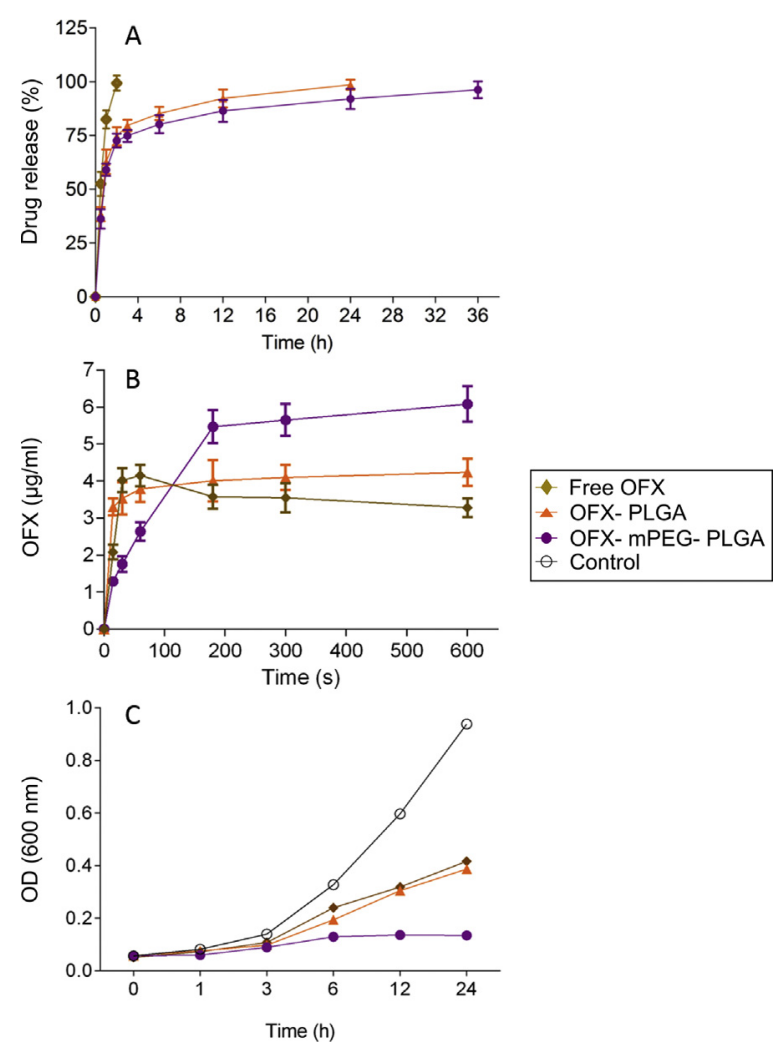

Fig. 3. (A) In vitro drug release profiles of free OFX and nanoformulations (OFX-PLGA and OFX-mPEG-PLGA). (B) Drug uptake of E. coli in the presence of free OFX and nanoformulations (OFX-PLGA and OFX-mPEG-PLGA). (C) Growth kinetics of E. coli cultured in the absence of drug (control) and in the presence of free OFX and its nanoformulations (OFX-PLGA and OFX-mPEG-PLGA). Data represents mean $\pm \mathrm{SD}, n$ $=3$.

coli cells is shown in Fig. 2D. Fluorescent images taken at various planes revealed that only a few bacterial cells internalized COUPLGA nanoparticles in a low frequency (Fig. 2E). Whereas, 95\% of bacterial cells have up taken COU-mPEG-PLGA nanoparticles in a higher frequency of 4-10 particles per bacterial cell (Fig. 2F). Collectively our results indicate that MPEG-PLGA nanoencapsulation is highly efficient in terms of bacterial uptake.

\subsection{Encapsulation efficiency, drug content and drug release profile}

Drug encapsulation efficiencies of OFX-PLGA and OFX-mPEGPLGA nanoparticles were $47 \%$ and 58\%, respectively. Estimation of drug content revealed that both OFX-PLGA and OFX-mPEGPLGA formulations contained $20 \mu \mathrm{g}$ OFX/mg nanoparticle. Drug release was significantly different between the free drug and naoparticulate forms $(P<0.05)$. Both nanoformulations showed an initial burst release up to $3 \mathrm{~h}$ and subsequent steady release (Fig. 3A). Although drug release was not significantly different between PLGA and MPEG-PLGA nanoparticles at the tested time points, the later showed an extended release. For instance, while OFX-PLGA nanoparticles released 99\% drug within $24 \mathrm{~h}$, OFXmPEG-PLGA nanoparticles released $96 \%$ of the encapsulated drug in $36 \mathrm{~h}$ (Fig. 3A).

\subsection{Intracellular drug concentration in $\mathrm{E}$. coli treated with free drug and drug-loaded nanoparticles}

Although OFX uptake was not statistically different between free drug and OFX-PLGA treated cultures until $300 \mathrm{~s}$, it was significantly different at $600 \mathrm{~s}(P<0.05)$. Drug uptake by bacterial cultures treated with OFX-mPEG-PLGA was significantly different from free drug and OFX-PLGA at all the time points tested $(P<0.05)$. In the case of free drug treatment, the maximum drug concentration $(4.15 \mu \mathrm{g} / \mathrm{ml}$ bacteria) was reached at $60 \mathrm{~s}$ post treatment and thereafter declined. Bacteria treated with OFX-PLGA attained a plateau of drug uptake ( $4.1 \mu \mathrm{g} / \mathrm{ml}$ bacteria $)$ in $300 \mathrm{~s}$. Interestingly, drug concentration in OFX-mPEG-PLGA treated bacteria reached $5.59 \mu \mathrm{g} / \mathrm{ml}$ in $300 \mathrm{~s}$ and still kept increasing (Fig. 3B).

\subsection{Growth kinetics of $\mathrm{E}$. coli in the presence of free drug and nanoparticles}

Up to $3 \mathrm{~h}$ post inoculation, the bacterial multiplication rate was more or less similar in control and treatments (OFX, OFX-PLGA and OFX-mPEG-PLGA). However, at $6 \mathrm{~h}$ a significant difference in the multiplication rate could be observed (Fig. 3C). Control cultures supplied with water or empty nanoparticles continued to multiply in an exponential phase reaching about $1.0 \mathrm{OD}$ at $24 \mathrm{~h}$. The bacterial multiplication rate in cultures treated with free drug and OFX-PLGA nanoparticle was more or less similar reaching an OD of 0.4 at $24 \mathrm{~h}$. Interestingly, cultures treated with OFX-mPEG-PLGA nanoparticles stayed almost unchanged throughout incubation, indicating stronger inhibition of bacterial multiplication by this nanoformulation.

\subsection{Antibacterial activity}

When the antibacterial activity of nanoparticles (OFX-PLGA and OFX-mPEG-PLGA) and free drug (OFX) was tested in clinically important human pathogenic bacteria (E. coli, P. aeruginosa, P. vulgaris, S. typhimurium, K. pneumoniae and S. aureus), free OFX showed the least antibacterial activity against all the tested bacteria (Fig. 4, Supplementary data S1). Compared to OFX-PLGA, OFX-mPEG-PLGA showed significantly higher antibacterial activity against all the tested bacteria. In the case of $S$. aureus, although OFX-mPEG-PLGA displayed higher potential than OFX-PLGA (Fig. 4), no statistical significance was observed (Supplementary data S1).

\subsection{Inhibition of bacterial resistance development in $\mathrm{B}$. subtilis}

Resistance development against OFX was analyzed in B. subtilis by zone inhibition assay (Fig. 5). At $24 \mathrm{~h}$ of incubation, the inhibition zone was higher in OFX-mPEG-PLGA treated wells $(22.8 \mathrm{~mm})$ than OFX-PLGA (15.0 mm) and free drug (19.2 mm). In spite of inhibition, tiny bacterial colonies could be seen inside the inhibition zone of OFX-PLGA (Fig. 5B), indicating that B. subtilis has already developed resistance against this treatment. On the other hand, the inhibition zones produced by OFX-mPEG-PLGA and free drug stayed clear by $24 \mathrm{~h}$ of incubation (Fig. $5 \mathrm{~A}$ and C). By $60 \mathrm{~h}$ of incubation, numerous colonies developed within the inhibition zones of free drug and OFX-PLGA treatment (Fig. 5D and E). Whereas, no colonies could be seen in the OFX-mPEG-PLGA inhibition zone even after prolonged incubation, witnessing its long lasting bactericidal effect and prevention of resistance development.

Fluorescent imaging of wells loaded with COU-PLGA and COU-mPEG-PLGA after $2 \mathrm{~h}$ showed efficient diffusion of these nanoparticles in the agar medium (Supplementary data S4).

\section{Discussion}

As in the case with any other antibacterial agents, the rise in quinolone resistance threatens the clinical utility of this important drug class [25]. Global surveillance studies demonstrates that the rate of fluoroquinolone resistance increased in the past years in almost all bacterial species [26,27], pointing to the imperative need 


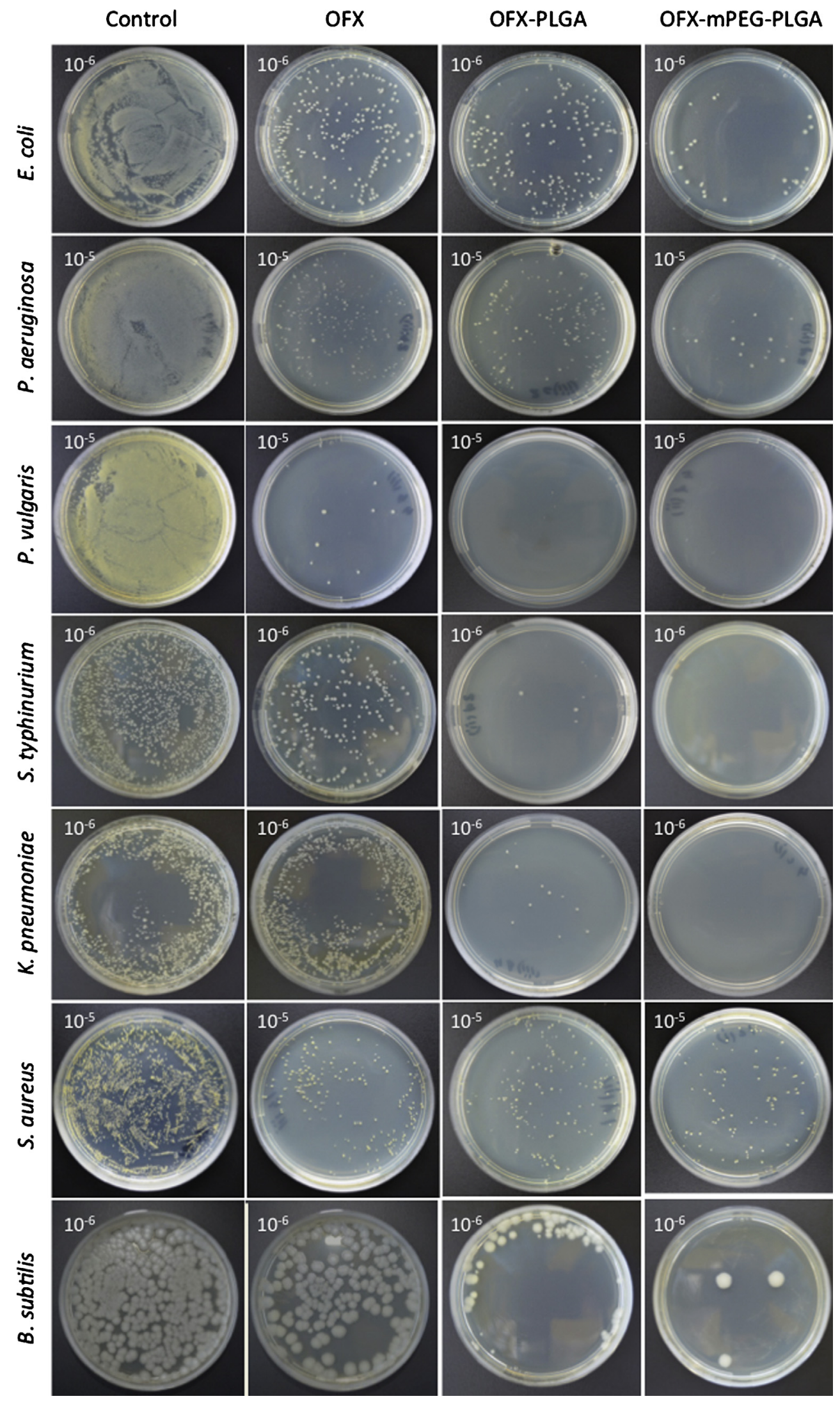

Fig. 4. Antibacterial activity of free OFX and nanoformulations. LB plates showing colony-forming units (CFU) of important human pathogens in the presence of free drug (OFX), drug loaded in PLGA (OFX-PLGA) and mPEG-PLGA (OFX-mPEG-PLGA) after 2 days of incubation at $37^{\circ} \mathrm{C}$. The dilutions of bacterial culture used for plating are represented in the left top corner of each figure. 


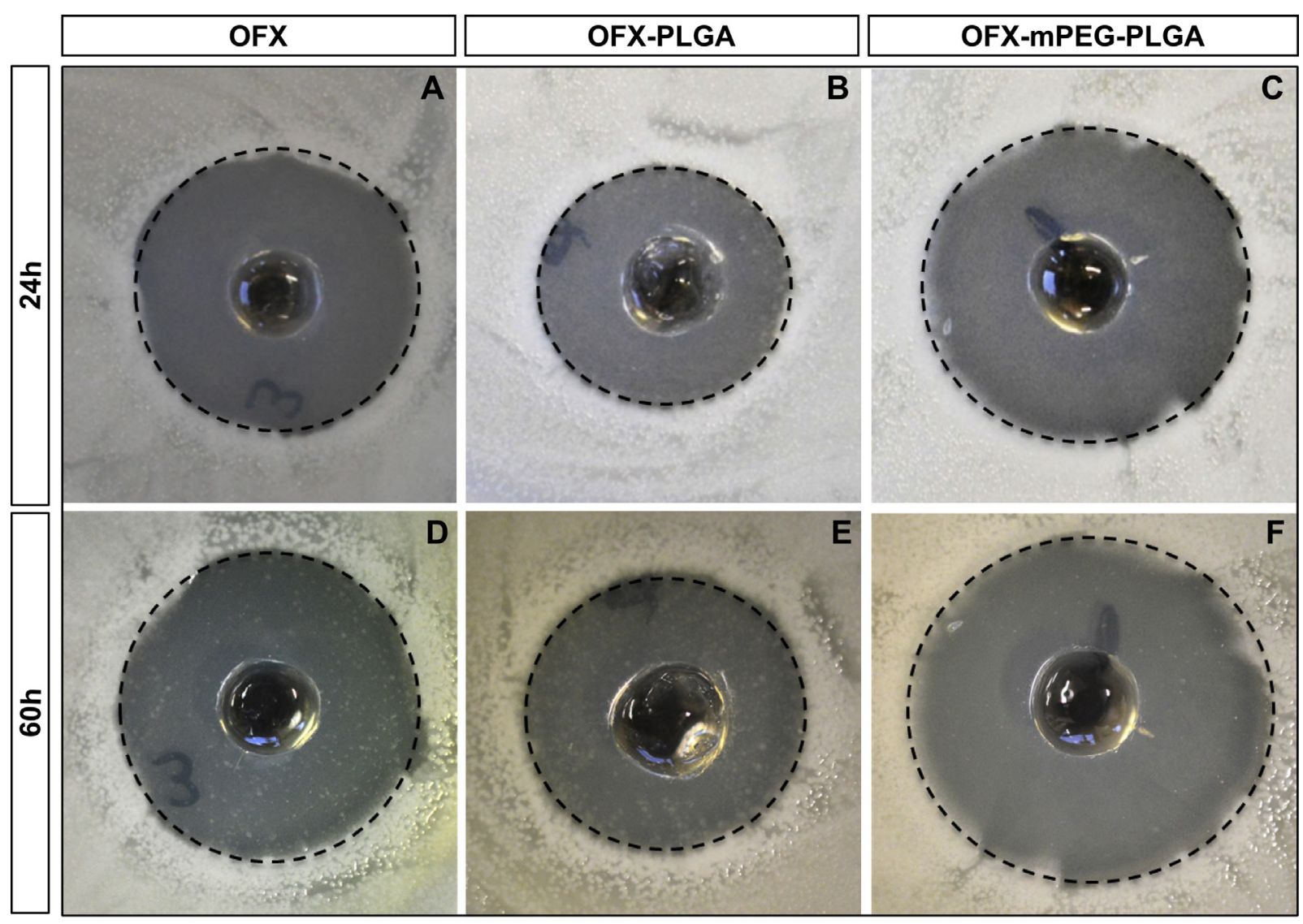

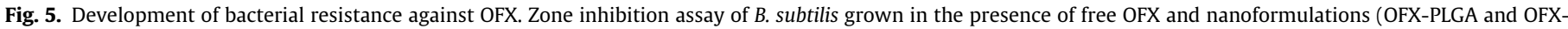
mPEG-PLGA). The margins of inhibition zone are indicated by dotted black circle.

for developing alternative strategies to avoid bacterial resistance. In this study, we have developed OFX-mPEG-PLGA nanoparticles that show higher antibacterial activity combined with an ability to inhibit bacterial resistance development, compared to OFXPLGA nanoparticles and free OFX. Along with efficient bacterial uptake (Fig. 2), sustained release (Fig. 3A) and strict control of bacterial growth (Figs. $3 \mathrm{C}$ and 4 ), we also attribute some unappreciated features of PEG for the enhanced activity of OFX-mPEG-PLGA nanoparticles.

In addition to the amphiphilic nature, smooth surface and uniform size of mPEG-PLGA nanoparticles (Fig. 1 and Supplementary data S2), one of the unnoticed features of PEG might also have facilitated its effective uptake by the bacteria. Much before its application in nanoformulations, PEG was used in bacterial/yeast transformations, where it presumably improves membrane permeability and thus bacterial competence to uptake foreign DNA from its surrounding environment [28]. In this context, PEGylation could have increased bacterial membrane permeability, allowing the accumulation of mPEG-PLGA nanoparticles inside the cells more than PLGA nanoparticles. Correspondingly, drug accumulation was also higher in bacterial cultures treated with OFX-mPEG-PLGA than OFX-PLGA nanoparticles (Fig. 3B). The increased uptake of drug-loaded mPEG-PLGA nanoparticles and subsequent drug accumulation should have contributed to the efficient antibacterial activity (Figs. 3C and 4). Accordingly, enhanced bacterial uptake of OFX loaded bioadhesive hydrogels or OFX-containing Eudragit E100 ${ }^{\circledR}$ dispersions were reported to increase bactericidal properties against $P$. aeruginosa $[29,30]$. In the present study, OFX-mPEGPLGA nanoparticles consistently showed better bactericidal activity against all the tested pathogenic bacterial strains, as revealed by the severe reduction in the number of colonies than the free drug or OFX-PLGA nanoparticles (Fig. 4 and Supplementary data S1).

Another uncredited property of PEG is its ability to bind DNA $[16,17]$ and this could also be accounted for the efficiency of OFX-mPEG-PLGA nanoparticles, because OFX encapsulated in these nanocarriers might have been delivered at the DNA, where it could act on the topoisomerases and DNA gyrase. In addition, higher encapsulation efficiency (10\% more than PLGA nanoparticles) and sustained release (Fig. 3A) of mPEG-PLGA nanoparticles should also be the reasons for its higher antibacterial efficacy.

Our results further showed that OFX-mPEG-PLGA nanoparticles were able to challenge the development of $B$. subtilis resistance compared to the free drug and OFX-PLGA nanoparticles (Fig. 5). In fact, extrusion of antibiotics by the membrane transporters called, efflux pumps are a widely accepted mechanism for bacterial drug resistance [31]. It should be noted that in B. subtilis multidrug efflux transporter bmr is reported to be responsible for clinically relevant resistance to fluoroquinolones [32] and it is also demonstrated that overexpression of $\mathrm{bmr}$ provided strong resistance to fluoroquinolones [33]. Since efflux pumps are a common mechanism utilized by both eukaryotic and prokaryotic cells to eliminate antibiotics [34,35], employing efflux pump inhibitor along with antibiotic is a wiser way to minimize the antibiotic extrusion. PEG has been previously shown to inhibit efflux pumps at the concentration of $0.1-20 \%$ [18]. Inhibition of efflux pumps by PEG 300 was shown to be mediated by changes in the microenvironment of Caco-2 cell membrane [19]. Thus, the improved antibacterial activity and minimized bacterial resistance of OFXmPEG-PLGA nanoparticles observed in our study might also be due to the fact that PEG is an efflux pump inhibitor. 


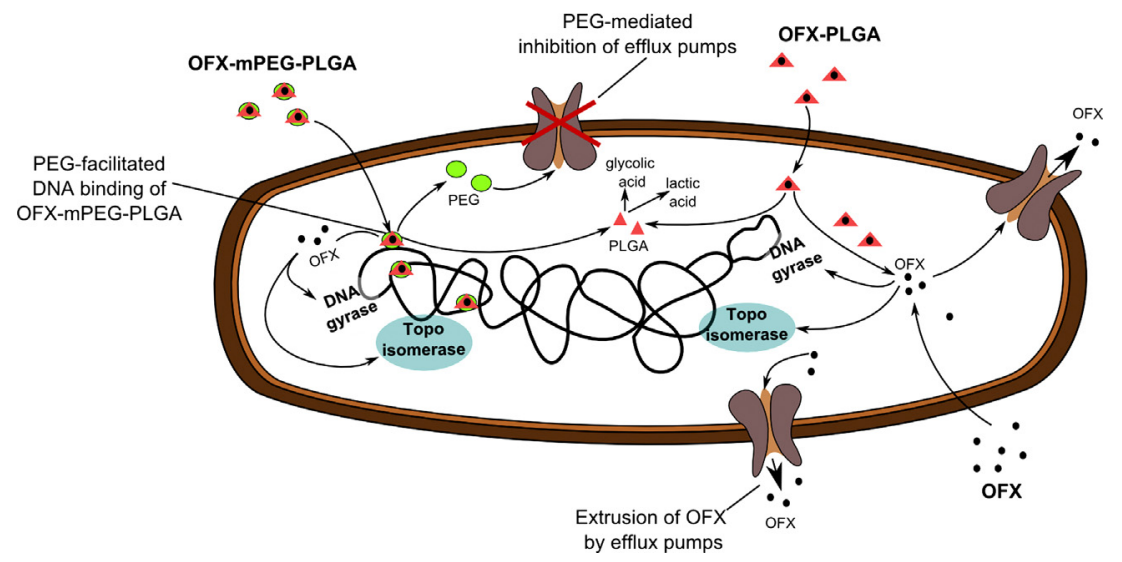

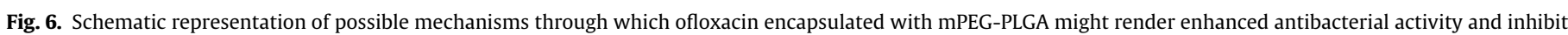
resistance development.

We have summarized our observations in association with previous reports in a form of a scheme, which may explain the efficiency of OFX-mPEG-PLGA over OFX-PLGA nanoparticles (Fig. 6). The membrane permeabilizing nature of PEG would facilitate enhanced entry of OFX-mPEG-PLGA nanoparticles inside the bacteria compared to the free dug and PLGA formulation. Once inside the bacteria, OFX-mPEG-PLGA nanoparticles could deliver OFX right at the site of action because of the DNA binding potential of PEG, which could have prevented the development of targeted mutations that generally contribute to resistance in B. subtilis. Moreover, hydrolytically cleaved mPEG from the nanoparticles could serve as efflux pump inhibitor, preventing the clearance of drug from the cell. Thus, the intracellular drug bound to DNA would act on the topoisomerases and DNA gyrase and inhibit bacterial DNA replication resulting in the arrest of bacterial multiplication. On the other hand, PLGA from the OFX-PLGA nanoparticles would have been degraded into lactic and glycolic acids that can be metabolized by the bacteria while releasing the drug in the cytoplasm, making it an easy target for extrusion by the efflux pumps (Fig. 6).

In conclusion, the PEGylated ofloxacin nanoparticle delivery system described in this study can be an interesting tool to treat local bacterial infections. PEGylation is commonly used to increase the circulatory time of the associated drug as it has been demonstrated for vancomycin in Murine model [36]. These authors showed that PEGylated liposomal encapsulation of vancomycin significantly prolonged its circulation time, compared to its standard and non-PEGylated formulations, improving its efficacy to treat pneumonia [36]. Since PEGylated nanoparticles can dramatically decrease opsonin adsorption and macrophage uptake due to the 'stealth' effect [37], PEGylated ofloxacin nanoparticles could be exploited for systemic administration as well.

\section{Funding}

G.M and C.J.S were supported by FCT - Fundação para a Ciência e a Tecnologia, Portugal, grants SFRH/BD/72809/2010 and SFRH/BPD/89493/2012, respectively. The present work was also supported by the FCT projects PTDC/AGR-GPL/119211/2010 and UID/AGR/04033/2013.

\section{Conflict of interest}

None declared.

\section{Ethical approval}

Not required.

\section{Appendix A. Supplementary data}

Supplementary data associated with this article can be found, in the online version, at http://dx.doi.org/10.1016/j.colsurfb.2015.04. 050

\section{References}

[1] A.S.S. Shaoo, R. Nanda, A.R. Phani, P.L. Nayak, Synthesis of chitosan-polycaprolactone blend for control delivery of ofloxacin drug, Carbohydr. Polym. 7 (2010) 106-113.

[2] J.P. Monk, D.M. Campoli-Richards, Ofloxacin. A review of its antibacterial activity, pharmacokinetic properties and therapeutic use, Drugs 33 (1987) 346-391.

[3] P.T. Djurdjevic, M. Jelikic-Stankov, Study of solution equilibria between aluminum(III) ion and ofloxacin, J. Pharm. Biomed. Anal. 19 (1999) 501-510.

[4] Y. Mizuki, I. Fujiwara, T. Yamaguchi, Pharmacokinetic interactions related to the chemical structures of fluoroquinolones, J. Antimicrob. Chemother. 37 (Suppl. A) (1996) 41-55.

[5] P.M. Furneri, M. Fresta, G. Puglisi, G. Tempera, Ofloxacin-loaded liposomes: in vitro activity and drug accumulation in bacteria, Antimicrob. Agents Chemother. 44 (2000) 2458-2464.

[6] H. Baskin, Y. Dogan, I.H. Bahar, N. Yulug, Effect of subminimal inhibitory concentrations of three fluoroquinolones on adherence of uropathogenic strains of Escherichia coli, Int. J. Antimicrob. Agents 19 (2002) 79-82.

[7] S.K.R. Namasivayam, K. Samrat, S. Ganesh, Preparation of chitosan stabilized ofloxacin-gold nano conjugate for the improved anti-bacterial activity against human pathogenic bacteria, Innovare J. Med. Sci. 1 (2013) 7-11.

[8] A. Dalhoff, Resistance surveillance studies: a multifaceted problem - the fluoroquinolone example, Infection 40 (2012) 239-262.

[9] K. Drlica, H. Hiasa, R. Kerns, M. Malik, A. Mustaev, X. Zhao, Quinolones: action and resistance updated, Curr. Top. Med. Chem. 9 (2009) 981-998.

[10] X.Z. Li, H. Nikaido, Efflux-mediated drug resistance in bacteria: an update, Drugs 69 (2009) 1555-1623.

[11] A. Beceiro, M. Tomas, G. Bou, Antimicrobial resistance and virulence: a successful or deleterious association in the bacterial world? Clin. Microbiol. Rev. 26 (2013) 185-230.

[12] E. Reilman, R.A. Mars, J.M. van Dijl, E.L. Denham, The multidrug ABC transporter $\mathrm{BmrC} / \mathrm{BmrD}$ of Bacillus subtilis is regulated via a ribosome-mediated transcriptional attenuation mechanism, Nucleic Acids Res. 42 (2015) 11393-11407.

[13] C.J. Sheeba, G. Marslin, A.M. Revina, G. Franklin, Signaling pathways influencing tumor microenvironment and their exploitation for targeted drug delivery, Nanotechnol. Rev. 3 (2) (2014) 123-151.

[14] N. Abed, P. Couvreur, Nanocarriers for antibiotics: a promising solution to treat intracellular bacterial infections, Int. J. Antimicrob. Agents 43 (2014) 485-496.

[15] G. Marslin, C.J. Sheeba, V.K. Kalaichelvan, R. Manavalan, P.N. Reddy, G. Franklin, Poly(D,L-lactic-co-glycolic acid) nanoencapsulation reduces Erlotinib-induced subacute toxicity in rat, J. Biomed. Nanotechnol. 5 (2009) 464-471.

[16] N. Elmarzugi, T. Adali, A. Bentaleb, E. Keleb, A. Mohamed, A. Hamza, Spectroscopic characterization of PEG-DNA biocomplexes by FTIR, J. Appl. Pharm. Sci. 4 (08) (2014) 6-10

[17] E. Froehlich, J.S. Mandeville, C.M. Weinert, L. Kreplak, H.A. Tajmir-Riahi, Bundling and aggregation of DNA by cationic dendrimers, Biomacromolecules 12 (2011) 511-517.

[18] B.M. Johnson, W.N. Charman, C.J. Porter, An in vitro examination of the impact of polyethylene glycol 400, pluronic P85, and vitamin E da-alpha-tocopheryl polyethylene glycol 1000 succinate on P-glycoprotein efflux and enterocytebased metabolism in excised rat intestine, AAPS Pharm. Sci. 4 (2002) E40.

[19] M. Werle, Natural and synthetic polymers as inhibitors of drug efflux pumps, Pharm. Res. 25 (2008) 500-511. 
70

G. Marslin et al. / Colloids and Surfaces B: Biointerfaces 132 (2015) 62-70

[20] P. Liu, H. Yu, Y. Sun, M. Zhu, Y. Duan, A mPEG-PLGA-b-PLL copolymer carrier for adriamycin and siRNA delivery, Biomaterials 33 (2012) 4403-4412.

[21] S. Mondal, P. Roy, S. Das, A. Halder, A. Mukherjee, T. Bera, In vitro susceptibilities of wild and drug resistant leishmania donovani amastigote stages to andrographolide nanoparticle: role of vitamin E derivative TPGS for nanoparticle efficacy, PLOS ONE 8 (2013) e81492.

[22] S. Hua, H. Yang, W. Wang, A. Wang, Controlled release of ofloxacin from chitosan-montmorillonite hydrogel, Appl. Clay Sci. 50 (2010) 112-117.

[23] M. Abazinge, T. Jackson, Q. Yang, G. Owusu-Ababio, Comparison of in vitro and in vivo release characteristics of sustained release ofloxacin microspheres, Drug Deliv. 7 (2000) 77-81.

[24] I.T. Paulsen, J. Chen, K.E. Nelson, M.H. Saier Jr., Comparative genomics of microbial drug efflux systems, J. Mol. Microbiol. Biotechnol. 3 (2001) 145-150.

[25] K.J. Aldred, R.J. Kerns, N. Osheroff, Mechanism of quinolone action and resistance, Biochemistry 53 (2014) 1565-1574.

[26] A. Dalhoff, Global fluoroquinolone resistance epidemiology and implications for clinical use, Interdiscip. Perspect. Infect. Dis. 2012 (2012) 976273.

[27] J. Davies, D. Davies, Origins and evolution of antibiotic resistance, Microbiol. Mol. Biol. Rev. 74 (2010) 417-433.

[28] C.T. Chung, S.L. Niemela, R.H. Miller, One-step preparation of competent Escherichia coli: transformation and storage of bacterial cells in the same solution, Proc. Natl. Acad. Sci. U.S.A. 86 (1989) 2172-2175.

[29] V.L. Romero, R.H. Manzo, F.L. Alovero, Enhanced bacterial uptake and bactericidal properties of ofloxacin loaded on bioadhesive hydrogels against Pseudomonas aeruginosa, J. Chemother. 22 (2010) 328-334.
[30] V.L. Romero, P. Pons, J.L. Bocco, R.H. Manzo, F.L. Alovero, Eudragit E100(R) potentiates the bactericidal action of ofloxacin against fluoroquinoloneresistant Pseudomonas aeruginosa, FEMS Microbiol. Lett. 334 (2012) 102-110.

[31] M.A. Fischbach, C.T. Walsh, Antibiotics for emerging pathogens, Science 325 (2009) 1089-1093.

[32] M. Ahmed, L. Lyass, P.N. Markham, S.S. Taylor, N. Vazquez-Laslop, A.A. Neyfakh, Two highly similar multidrug transporters of Bacillus subtilis whose expression is differentially regulated, J. Bacteriol. 177 (1995) 3904-3910.

[33] M.M.S. Kumar, F.M. Varela, Modulation of bacterial multidrug resistance efflux pumps of the major facilitator superfamily, Int. J. Bacteriol. (2013) 1-15.

[34] F. Van Bambeke, Y. Glupczynski, P. Plesiat, J.C. Pechere, P.M. Tulkens, Antibiotic efflux pumps in prokaryotic cells: occurrence, impact on resistance and strategies for the future of antimicrobial therapy, J. Antimicrob. Chemother. 51 (2003) 1055-1065.

[35] F. Van Bambeke, J.M. Michot, P.M. Tulkens, Antibiotic efflux pumps in eukaryotic cells: occurrence and impact on antibiotic cellular pharmacokinetics, pharmacodynamics and toxicodynamics, J. Antimicrob. Chemother. 51 (2003) 1067-1077.

[36] K. Muppidi, J. Wang, G. Betageri, A.S. Pumerantz, PEGylated liposome encapsulation increases the lung tissue concentration of vancomycin, Antimicrob. Agents Chemother. 55 (2011) 4537-4542.

[37] S. Xie, Y. Tao, Y. Pan, W. Qu, G. Cheng, L. Huang, D. Chen, X. Wang, Z. Liu, Z. Yuan, Biodegradable nanoparticles for intracellular delivery of antimicrobial agents, J. Control. Release 187 (2014) 101-117. 